\title{
Comparison of morphological changes in efferent lymph nodes after implantation of resorbable and non-resorbable implants in rabbits
}

\author{
Alexandr Bondarenko ${ }^{3}$, Marion Hewicker-Trautwein ${ }^{2}$, Nina Erdmann ${ }^{1}$, Nina Angrisani ${ }^{1}$, Janin Reifenrath ${ }^{1 *}$ and \\ Andrea Meyer-Lindenberg ${ }^{4}$
}

\footnotetext{
* Correspondence: janin. reifenrath@tiho-hannover.de ${ }^{1}$ Small Animal Clinic, University of Veterinary Medicine Hannover, Bünteweg 9, 30559 Hannover, Germany

Full list of author information is available at the end of the article
}

\begin{abstract}
Background: Magnesium alloys as biodegradable implant materials received much interest in recent years. It is known that products of implant degradation can induce several types of immune response. Hence, the aim of this study was to examine the morphological changes of efferent lymph nodes after implantation of different resorbable magnesium alloys (MgCa0.8, LAE442) in comparison to commercially available resorbable (PLA) and non-resorbable (titanium) implant materials as well as control groups without implant material.

Methods: The different implant materials were inserted intramedullary into the rabbit tibia. After postoperative observation periods of three and six months, popliteal lymph nodes were examined histologically and immunhistologically and compared to lymph nodes of sham operated animals and animals without surgery. Haematoxylin and eosin staining was performed for cell differentiation. Mouse antiCD79 $\alpha$ and rat anti-CD3 monoclonal primary antibodies were used for B- and Tlymphocyte detection, mouse anti-CD68 primary antibodies for macrophage detection. Evaluation of all sections was performed applying a semi quantitative score.

Results: The histological evaluation demonstrated low and moderate levels of morphological changes for both magnesium alloys (LAE442 and MgCa0.8). Higher than moderate values were reached for titanium in sinus histiocytosis and histiocytic apoptosis ( 3 months) and for PLA in histiocytic apoptosis ( 3 and 6 months). The immune response to all investigated implants had a non-specific character and predominantly was a foreign-body reaction. LAE442 provoked the lowest changes which might be due to a lower degradation rate in comparison to MgCa0.8.

Therewith it is a promising candidate for implants with low immunogenic potential.

Conclusion: Both examined magnesium alloys did not cause significantly increased morphological changes in efferent lymph nodes in comparison to the widely used implant materials titanium and PLA. LAE442 induced even lower immunological reactions. Therewith MgCa0.8 and especially LAE442 are appropriate candidates for biomedical use.
\end{abstract}




\section{Background}

Nowadays there are many studies dedicated to the research of biodegradable implants' influence on living tissues [1-8]. An important aim of these studies is the investigation of immunological effects of biodegradable materials [9-18].

Degradation products of metallic biomaterials include particulate wear debris, colloidal organometallic complexes (specifically or non-specifically bound), free metallic ions, inorganic metal salts or oxides and precipitated organometallic storage forms [9]. If the different substances with a various biochemical activity are present in a local area, the metal implants may influence the immune system in different possible ways: immune response mediated by type IV delayed hypersensitivity (DTH) $[9,10,17,18]$, immune suppression via apoptosis of responsible cells [19-22] and foreign-body reaction [5].

Even in spite of chemical inertness of metals like titanium, corrosion processes in contact with biological systems (aging of prosthesis) are described [23,24], accompanied by release of ions, which are not sensitizers on their own, but can induce the immune system by generating complexes with native proteins [10-14,25-27]. These metal-protein complexes are supposed to be candidate antigens (i.e. allergens) for developing delayed hypersensitivity [11]. DTH based on interactions between antigen-presenting cells, which process and present antigen, and CD4+ T-cells, which initiate this type of immune response by the release of cytokines and by macrophage activation [9].

Candidate antigen-presenting cells in the periimplant region include macrophages, endothelial cells, Langerhans cells, dendritic cells and to lesser extent parenchymal tissue cells [11]. The clonal T-lymphocyte specificity associated with type IV delayed hypersensitivity remains the dominant mechanism associated with implant related hypersensitivity responses [11-13].

Resorbable implant materials, inclusive magnesium containing alloys, have been investigated as sources of hypersensitivity-type immune responses. Witte et al. [3] demonstrated the absence of skin sensitizing properties for standard implant materials PLA (SR-PLA96) and titanium (TiAl6V4) as well as for the investigated magnesium alloys (AZ31, AZ91, WE43, and LAE442). However, immunogenic reactions associated with polymers have been reported, albeit less frequently [15]. Additionally, the sensitizing properties of different metals (haptenic components in antigens) are described [16]. Also, occasional responses to titanium have been demonstrated $[17,18]$.

The induction of apoptosis, that can reflect the immunosuppressive activity of implants, was reported for magnesium and calcium [28-30], rare earth elements [28], titanium particles $[19,20,23]$ and PLA [22].

Inflammatory host responses to different resorbable implant materials in local area have been well described in several studies $[1,4,5]$. Implantation of PLA as well as magnesium-containing resorbable materials induced the insignificant non-specific inflammation in surrounding tissues and demonstrated a moderate level of neutrophilic and macrophage infiltration, presence of giant foreign-body cells and small amount of Tlymphocytes with a decrease tendency of these parameters from three to six months of implantation period $[4,5]$. Unfortunately, just a few of contemporary studies described morphological changes in the regional lymph nodes after the intraosseous implantation of PLA [31] and in spleen after the drainage of different metal ions [32], though it is well known that any inflammatory stimuli involve the lymph nodes, which act as 
defensive barriers [33]. Thus, any immune response against foreign antigens is often associated with lymph node enlargement (lymphadenopathy) [33]. This condition can assume one of three patterns, depending on the causative agent: follicular hyperplasia, which is associated with B-cell activation, paracortical hyperplasia that shows reactive changes within the T-cell regions or sinus histiocytosis [33]. Additionally, some researchers reported the cases of sinus histiocytosis in regional lymph nodes induced by the implantation of prostheses contained chromium, cobalt and titanium [34,35].

Hence, in consideration of current data, an evaluation which could reflect the real condition of local and systemic immunity in patients with biodegradable implants and which would allow evaluating the host reaction is missing. Moreover, the appearance of such new biodegradable materials as magnesium-calcium alloys or magnesium-rare earth metal alloys [1,36-38] combined with deficiency of data about their immunogenic properties determines the actuality and necessity of the study of morphological changes in lymphoid tissue under the influence of these materials.

The aim of this study was to compare changes in popliteal lymph nodes after tibial bone implantation of magnesium alloys with standard implant materials in a rabbit model using histological and immunohistochemical techniques.

\section{Methods}

For this study, the popliteal lymph nodes as regional drainage collectors of animals with the implanted magnesium-based alloys MgCa0.8 and LAE442 were examined in comparison to the established materials PLA (resorbable) and titanium (non-resorbable) as well as control groups without implant material.

\section{Implant material}

The magnesium alloy $\mathrm{MgCa} 0.8$ contains adjacent to magnesium $0.8 \mathrm{wt} \%$ calcium, the alloy LAE442 the elements lithium (4 wt\%), aluminium (4 wt\%) and a rare earth composition metal ( $2 \mathrm{wt} \%)$. Main fractions in the composition metal were cerium (1.3 wt $\%)$, neodymium (0.37 $\mathrm{wt} \%$ ) and lanthanium (0.5 wt\%). The alloy designation for LAE442 is in accordance with the ASTM-standard (American Society for Testing Materials). The alloy designation for $\mathrm{MgCa} 0.8$ is based on the glossary of chemical elements. The extruded magnesium implants of $25 \mathrm{~mm}$ length and $2.5 \mathrm{~mm}$ in diameter were produced according to previous studies [2,6]. Titanium implants (TiAl6V4, 25 $\mathrm{mm}$ length and $2.5 \mathrm{~mm}$ in diameter, Synthes, Germany) and PLA implants (poly-96L/ 4D-lactide, $25 \mathrm{~mm}$ length and $2.0 \mathrm{~mm}$ in diameter, Synthes, Germany) were commercially available.

\section{Animal Model}

The animal experiment was authorized according to the German Animal Welfare Act and registered as number 509.6-42502/3-04/750. All materials were implanted intramedullary into both tibiae of adult, female New Zealand White rabbits (average weight 3.5 $\mathrm{kg}$ ) with implantation periods of three and six months. Five rabbits were used for each group containing magnesium alloys, two rabbits were used for the PLA-group and two for the titanium-group.

As control groups, three rabbits in each time group received the same surgical procedure without pin implantation. An intramedullary trauma was caused with a plastic 
pestle (sham operated group) to differentiate a postoperative local inflammation from an implant induced inflammatory reaction. Six lymph nodes of animals which were used in other studies without surgery on the limb served as control for physiological lymph node morphology (control group).

Anaesthesia, surgical procedure and euthanasia as well as the examination of the boneimplant compound were described in detail by Krause et al. [2] and Thomann et al. [6].

\section{Histological and immunohistochemical setup}

One popliteal lymph node of each group was explanted, fixed in $4 \%$ formalin ( $48 \mathrm{~h}$, room temperature) and routinely embedded in paraffin.

Sections of 2-3 $\mu \mathrm{m}$ thickness were performed using a microtome (RM2255, Leica, Germany). Every explored lymph node was cut in 2-3 subsequent slices with interruption in 2-6 $\mu \mathrm{m}$. For histological studies, the sections were stained with haematoxylin and eosin $(\mathrm{H} \& \mathrm{E})$ and immunohistochemistry, which were performed according to established methods [39]. Antigen exposure was realised by heat-induced antigen retrieval (HIAR) method in citrate buffer ( $\mathrm{pH}$ 6.0). Non-specific binding was reduced by incubation with normal goat serum (1:5) diluted in phosphate-buffered saline (PBS, pH 7.1) for 20 min at RT. Mouse anti-CD79 $\alpha$ (HM 47/A9, Acris, Germany) for B-celldetection, rat anti-CD3 (clone CD3-12, Serotec, Germany) for T-cell-detection and mouse anti-CD68 (EBM11, Dako, Germany) for the detection of histiocytes (macrophages) were used as the primary antibodies, which were diluted in PBS containing bovine serum albumin (BSA, 1\%). The reactivity has been already tested on the rabbit animal model [39]. As secondary antibodies goat anti-mouse (biotinylated anti-mouse IgG $(\mathrm{H}+\mathrm{L})$, Vector Labs, Burlingame, $\mathrm{CA})$ and goat anti-rat biotin-conjugated IgG (Biotinylated Anti-Rat IgG $(\mathrm{H}+\mathrm{L})$, Vector Labs, Burlingame, $\mathrm{CA})$ was applied in concentration 1:200. Vectastain Elite ABC Kit (Biozol Diagnostica, Germany) was utilised as visualisation system. Diaminobenzidine (DAB) served as chromogen. The background was stained with Meyer's haemalaun. Negative control sections, in which the antibody was replaced by PBS, were included in all staining runs.

\section{Histological evaluation and scoring system}

All sections were observed by light microscopy (Axio Imager Z1, Zeiss, Germany). The pictures were edited with Axio Vision imaging program (version 4.7.1).

After a three times repeated evaluation of all sections, a specific set of the most abundant changes was established. Four of the most common characteristics (sinus histiocytosis, follicular hyperplasia, heterophilic infiltration and histiocytic apoptosis) were chosen. In H\&E stained sections, heterophiles were counted. Sinus histiocytosis and follicular hyperplasia were evaluated semiquantitatively as a comparison of follicles and sinuses in lymph nodes of experimental groups with the size of appropriate compartments (follicles and sinuses respectively) of intact popliteal lymph nodes in the control group of rabbits without surgery (Table 1). The presence of B-cells, T-cells and histiocytes in H\&E staining was confirmed by CD79 $\alpha$, CD3 and CD68 immunostaining. Apoptotic changes of histiocytes were detected in appearance of karyopyknosis, karyorrhexis (Figure 1) and, seldom, apoptotic bodies [21]. The histiocytes with indicated apoptotic changes were counted and evaluated in accordance to the scoring system (Table 1). 
Table 1 Scoring system for the histological evaluation of different pathological changes in the lymph nodes

\begin{tabular}{ccccc}
\hline Score & \multicolumn{2}{c}{ Cell number per field of view } & \multicolumn{2}{c}{ Level of compartment enlargement } \\
& heterophils & apoptotic histiocytes & sinus histiocytosis & follicular hyperplasia \\
\hline 0 & 0 & 0 & - & - \\
1 & $1-15$ & $1-5$ & + & + \\
2 & $15-25$ & $5-10$ & ++ & ++ \\
3 & $26-35$ & $11-15$ & +++ & +++ \\
4 & $36-45$ & $16-20$ & ++++ & ++++ \\
5 & $>45$ & $>20$ & +++++ & +++++ \\
\hline
\end{tabular}

\section{Statistics}

The mean values of the semiquantitative scoring were tabulated and differences between the material and time groups statistically analysed using a Mann-Whitney U test (SPSS for Windows). $\mathrm{P}<0.05$ indicated statistical significance.

\section{Results}

The most common morphological change in all investigated material groups was sinus histiocytosis, followed by heterophilic infiltration, appearance of histiocytic apoptosis, and rarely, occurrence of follicular hyperplasia. Distribution of these features in lymph nodes after exposure to different implant materials was not equally.

The control groups with surgery but without implant showed the presence of sinus histiocytosis, follicular hyperplasia and heterophilic infiltration with low score values overall and demonstrated decrease of all investigated characteristic values from three to six months. The control group without surgery had the lowest score values in all investigated parameters. Expression of CD3 and CD79 $\alpha$ in physiological (Figure 2B, $2 \mathrm{C}$ ) as well as in affected lymph nodes was conformed to $\mathrm{T}$ - and B-dependent zones (Figure 3C, 3E). CD3 was expressed in T-cells of the paracortical zone as clear membrane staining. Single T-cells were found in cortex near or within the follicles. There were no significant differences for CD3 expression in all groups and times including the control group without surgery (Figure 2C). CD79 $\alpha$ was expressed in B-cells of the

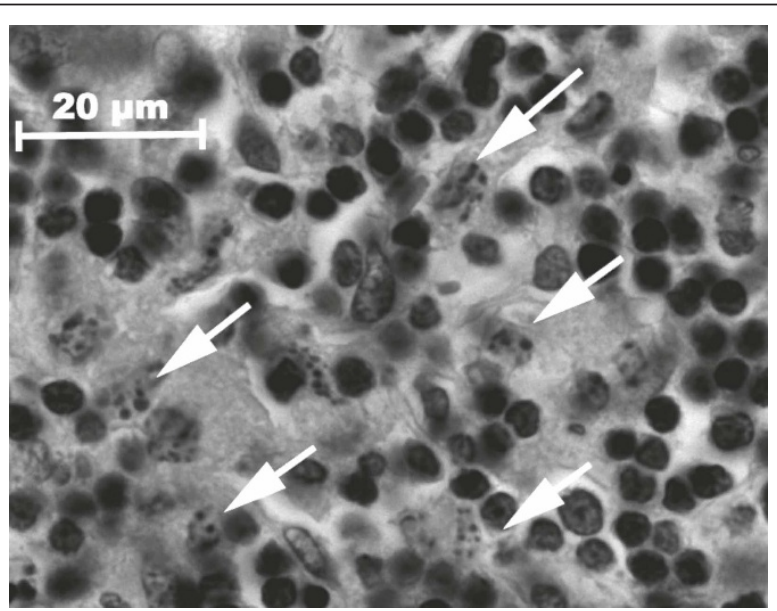

Figure 1 Histiocytic karyorrhexis (arrows) as sign for apoptotic changes of histiocytes, (H\&E, $\times$ 1000). Histiocytes with indicated apoptotic changes were counted and evaluated in accordance to the scoring system. 


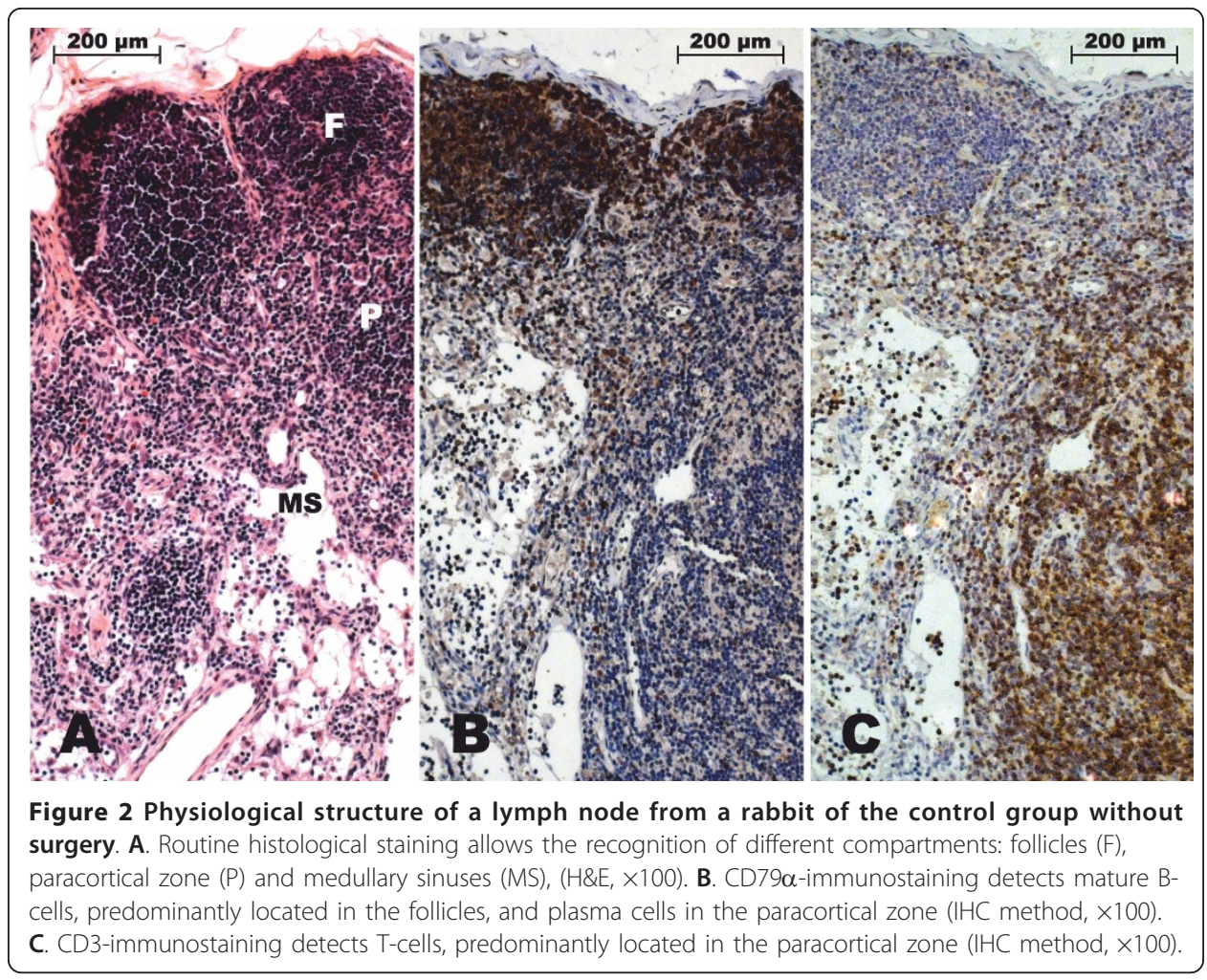

cortex, primary (Figure 2B) and secondary follicles, and rarely in medullary cords (plasma cell reaction). A few of B-cells and plasma cells were found in sinuses.

\section{Sinus histiocytosis}

The most widespread change in all groups was sinus histiocytosis except the control group without surgery which showed a low score value (SV 1.3). This reactive pattern is characterized by distension and prominence of the lymphatic sinusoids, due to a

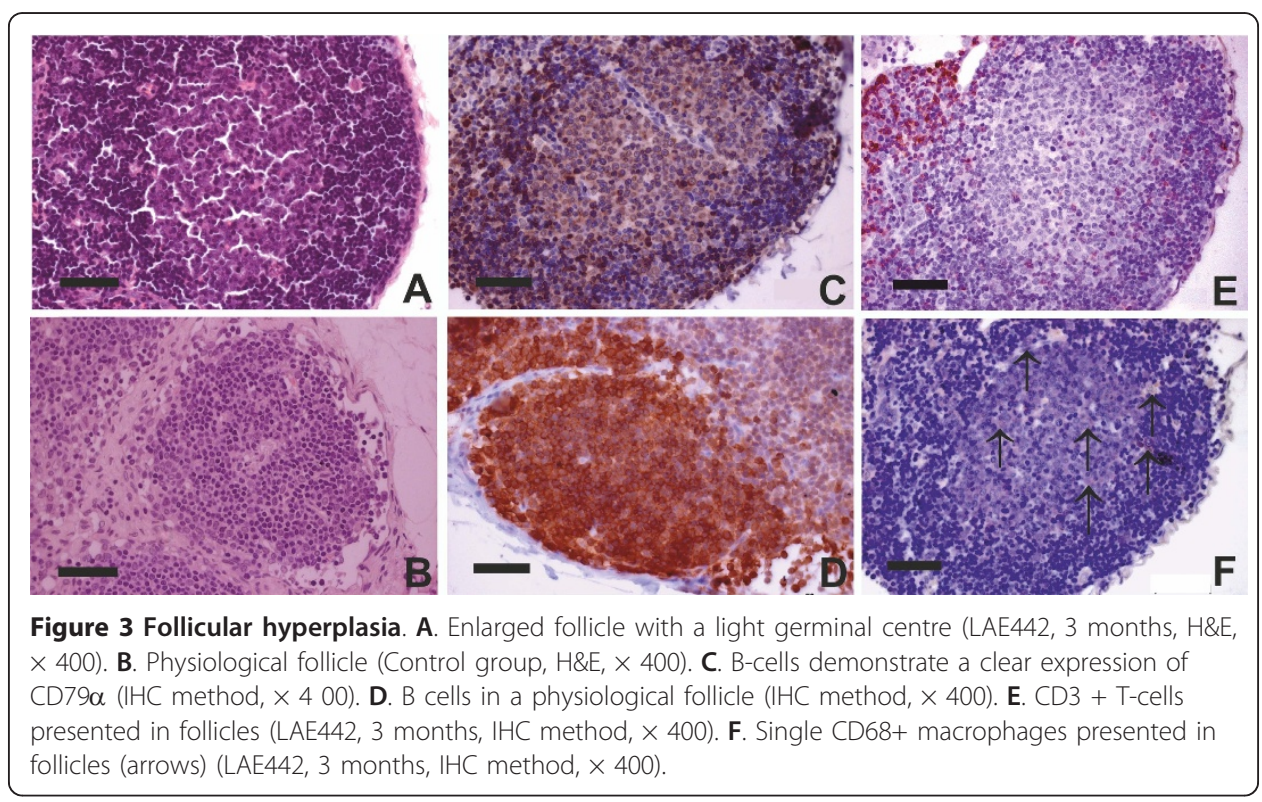




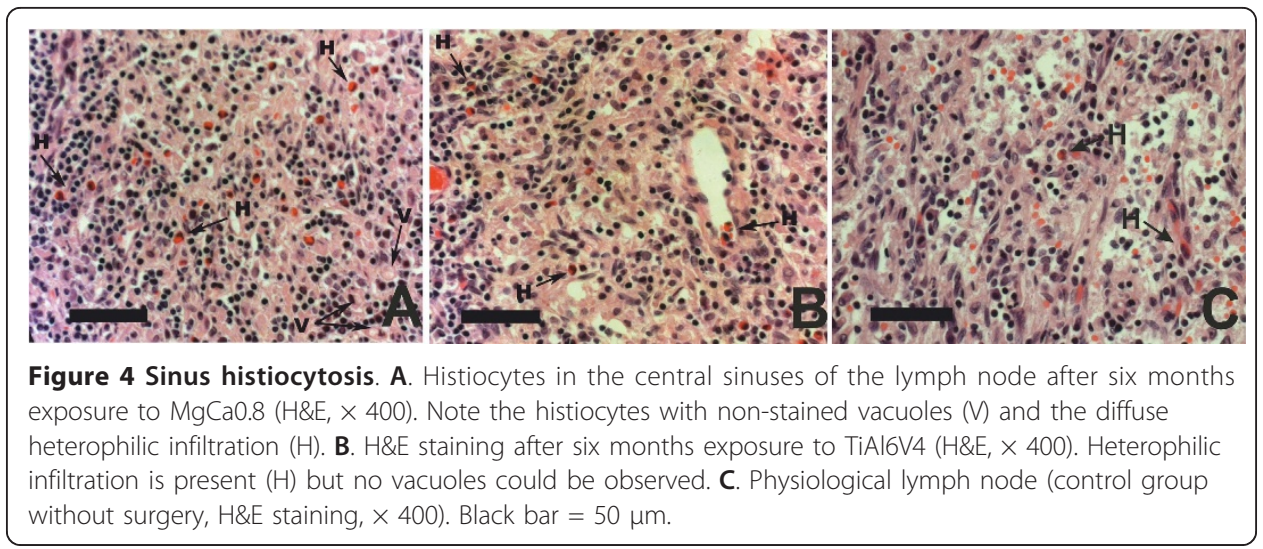

marked hypertrophy of lining endothelial cells and an infiltration of histiocytes. Numerous histiocytes in lymph nodes from both groups with magnesium-based implants (MgCa0.8 and LAE442) were found with foamy cytoplasm and non-stained vacuoles which contained small pale basophilic particles (Figure 4A). Histiocytes were recognized as CD68-positive cells (Figure 5) and were located in central and marginal sinuses, though single cells occurred within the cortical and paracortical zones (Figure 3F). After three months implantation duration, the titanium group showed the highest score values for sinus histiocytosis (score value 4), which were significant higher than both magnesium based alloys, the sham operated group and the control without surgery (MgCa0.8 SV 2.6, LAE442 SV 2.2, sham operated SV 2.5, control without surgery SV 1.3, $\mathrm{p}<0.05)$. The PLA-group demonstrated a moderate level of this parameter (SV 3.0). While the score values for titanium and PLA decreased from three to six months (Ti, SV 2.7 and PLA, SV 2.3), the value for MgCa0.8 increased insignificantly (SV 3.0) so that it exceeded those of PLA and titanium. LAE442 showed the lowest score values of all groups with implant material after both implantation periods with a few tendency of decrease over the time (SV 2.2 and 2.0), very similar to the sham operated group (SV 2.5 and 1.9) (Figure 6).

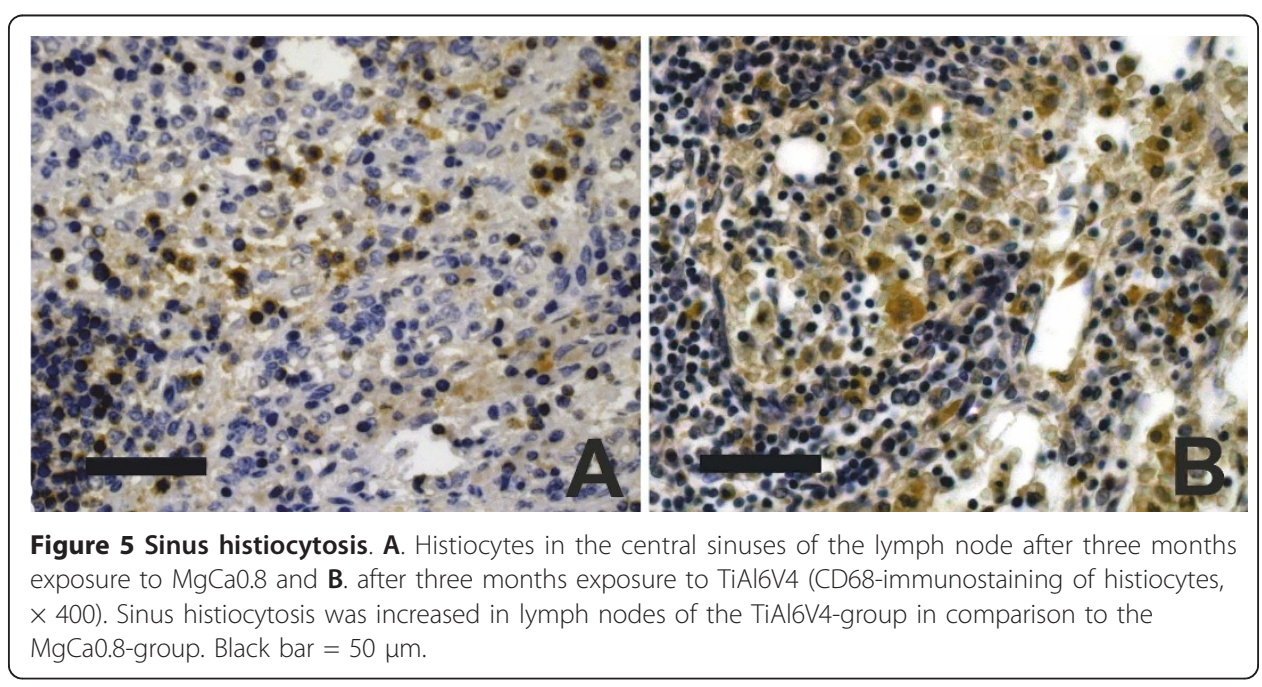




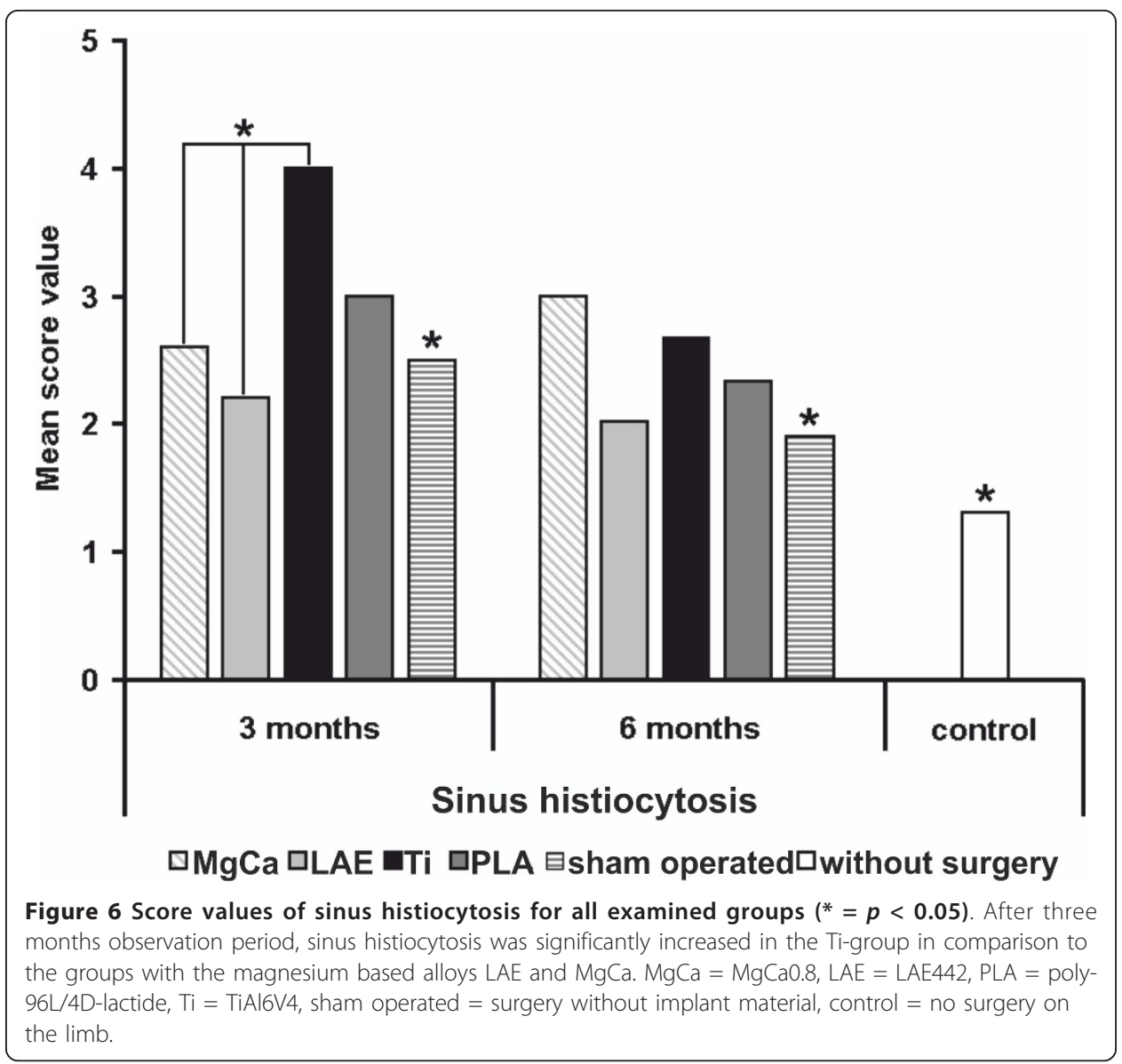

\section{Apoptosis of histiocytes}

The most distinct differences between the material groups could be found with the evaluation of histiocytic apoptosis. After three months implantation duration PLA (SV 4.0) and titanium (SV 3.5) showed significant higher values $(\mathrm{p}<0.05)$ than $\mathrm{MgCa} 0.8$ (score 0.8), with the lowest level of apoptosis, LAE442 (score 1.8) and both control groups (sham operated SV 1.0 and control without surgery SV 0.3). After six months of implantation duration the results changed obviously. $\mathrm{MgCa} 0.8$ showed a distinct increase in the number of apoptotic histiocytes (score value 3.0, p < 0.05). Contrary, the values for PLA (SV 3.7), titanium (SV 2.7), LAE442 (SV 1.6) and for the sham operated group (SV 0.7) decreased. LAE442 showed clearly lower score values than all other material groups (Figure 7).

\section{Heterophilic infiltration}

Heterophils were found in sinuses (Figure 4) and, rarely, in follicles and the paracortical zone. After three months of implantation duration, MgCa0.8, PLA and the sham operated group showed higher score values for heterophilic infiltration (SV 2.6, SV 3.0 and SV 2.7) than LAE442 and titanium (both SV 2.0) as well as the control group without surgery (SV 1.3). While the score increased for MgCa0.8 (SV 2.8) and thus showed the highest values of all materials after six months the score value for PLA decreased (SV 2.3) and reached a similar level as titanium (SV 2.3). LAE442 (SV 1.8) 


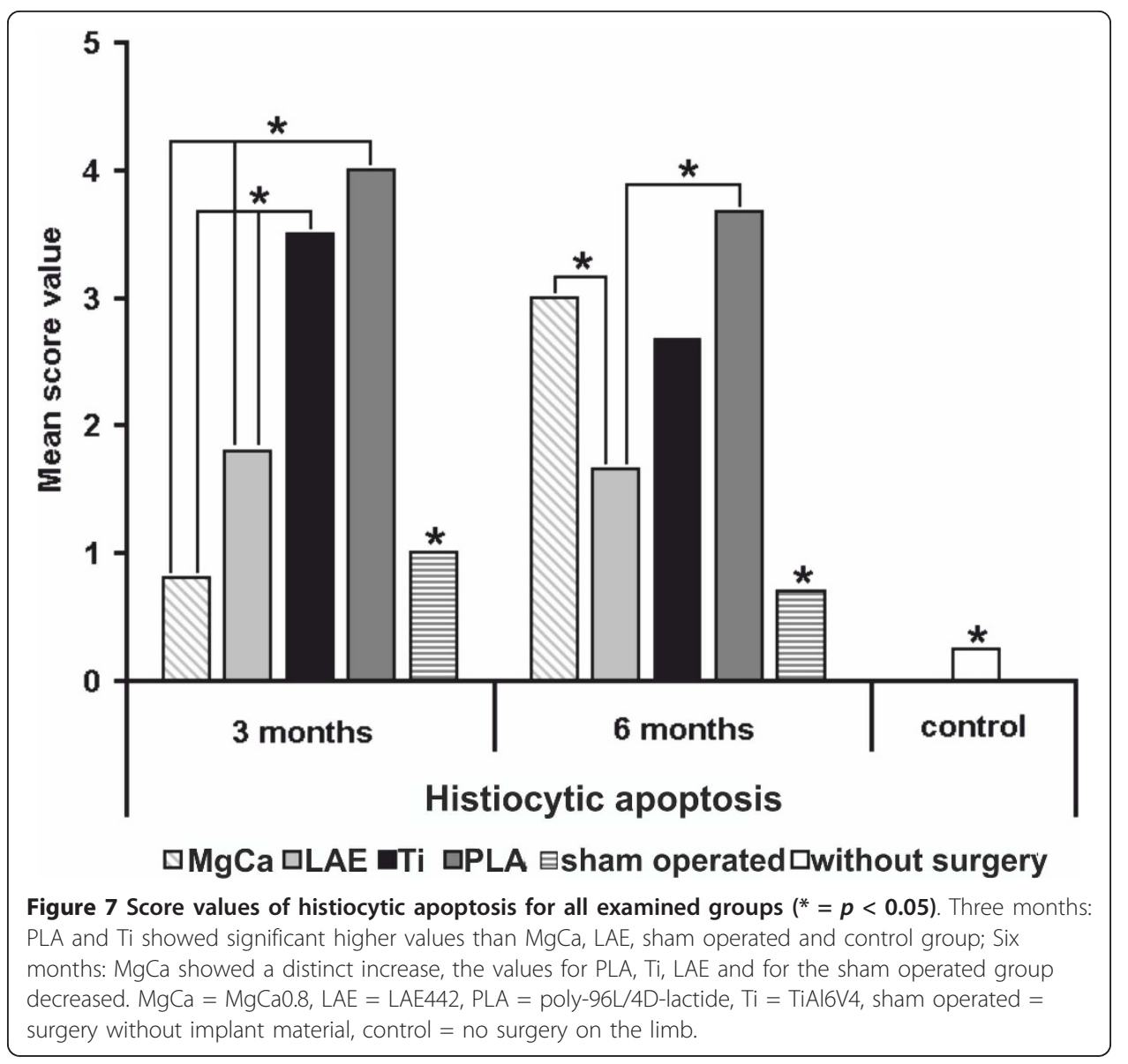

showed the lowest level of all tested materials, even lower than the sham operated group (SV 2.0) after six months implantation duration. Described differences were not statistically significant between the investigated groups (Figure 8). However, they were significantly higher than in the control group without surgery.

\section{Follicular hyperplasia}

The lymph nodes from the control group without surgery did not show any signs of follicular hyperplasia (SV 0.0). After three months implantation duration, follicular hyperplasia was found in a moderate level for all material groups (LAE442, SV 2.2; PLA and Ti, SV 2.0; MgCa0.8, SV 1.6) as well as in the sham operated group (SV 1.5). After six months, a significant decrease could only be seen in the control group without surgery (SV 0.5), a slight decrease was found in LAE442 (SV 1.9). The other material groups showed no difference over time (PLA, SV 2.0) or a slight increase (MgCa0.8, SV 1.8) (Figure 9). The increase of CD79 $\alpha$ expression was corresponded to the presence of follicular hyperplasia in H\&E-staining (Figure 3). However, as was mentioned above, there were no meaningful differences between all groups and times.

\section{Discussion}

The presented study was performed to demonstrate and compare the morphological changes in peripheral lymphoid tissue, which can reflect the condition of local immunity after the exposure to commonly used (PLA) and newly developed (MgCa0.8 and 


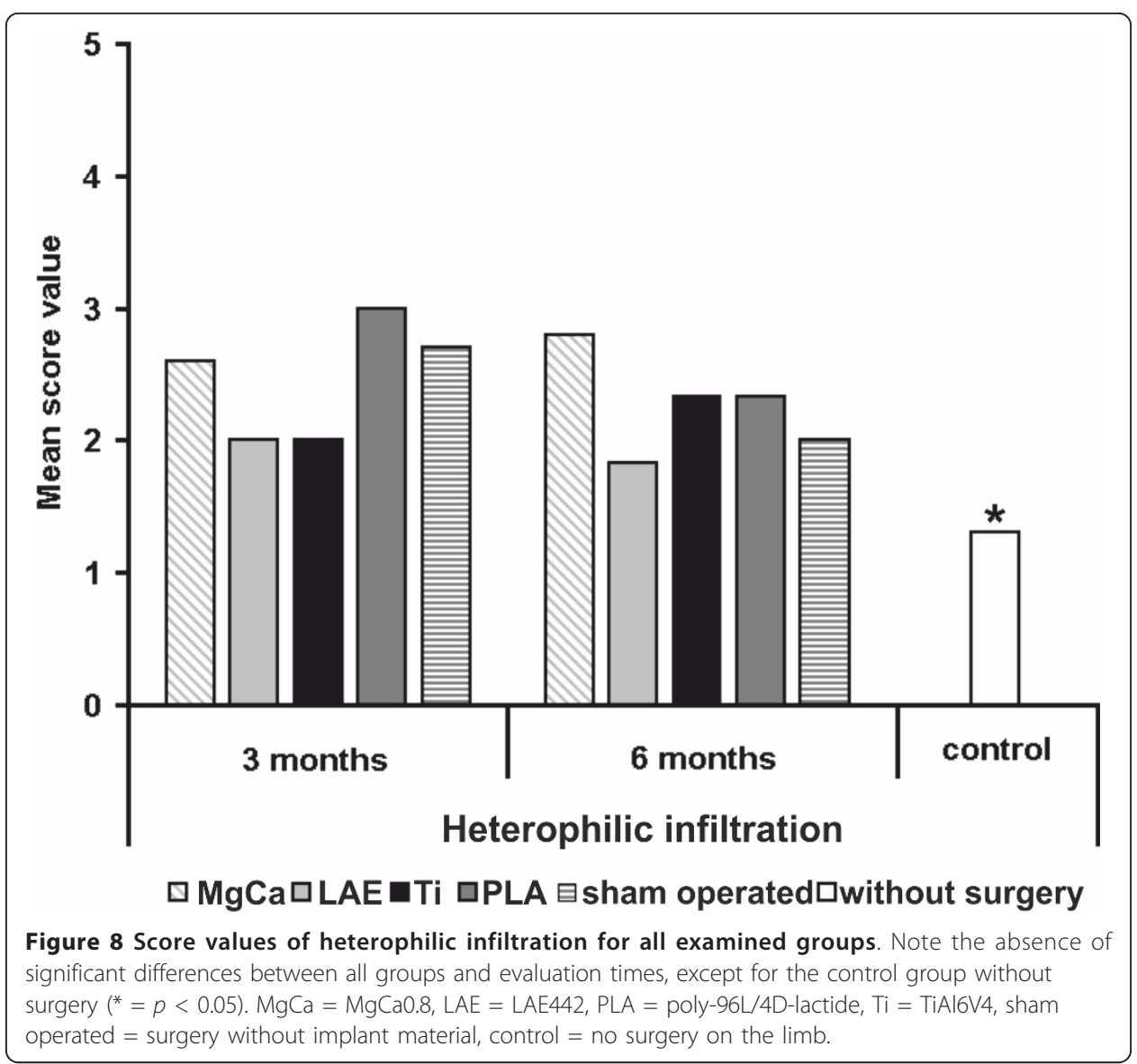

LAE442) resorbable and non-resorbable (titanium) bone implants in comparison to control groups. The results show, that the four examined implant materials as well as the surgical procedure itself provoke similar reactions within the regional lymphoid tissue, which were sinus histiocytosis, follicular hyperplasia, heterophilic infiltration and apoptosis of histiocytes. The changes in efferent lymph nodes in all material groups after observation periods of three and six months were mainly moderate without significant differences in comparison to the control groups with surgery for heterophilic infiltration and follicular hyperplasia. Higher than moderate values were only demonstrated in the commonly used materials titanium, for sinus histiocytosis and apoptosis of histiocytes (titanium, 3 months), and PLA, for sinus histiocytosis (PLA, 3 and 6 months). Lee et al. investigated the release of metal elements from titanium implants [40]. Titanium particles were neither detected in periimplant soft tissue nor in regional lymph nodes. However, it cannot be excluded that titanium implants induce an immuno-inflammatory response leading to reactions in regional lymph nodes.

The presence of sinus histiocytosis, follicular hyperplasia and heterophilic infiltration in the examined lymph nodes could be explained by different but joined ways of immune reaction. Follicular hyperplasia develops in lymph nodes which react to inflammatory processes in the area they filter. The offending antigen is brought into the lymph node by lymphatic drainage, where it is phagocytized and degraded within macrophages, and therewith initiates an immune reaction by activating B-cells, which enter into B-cell follicles and create the reaction of a germinal centre [33]. The rare 


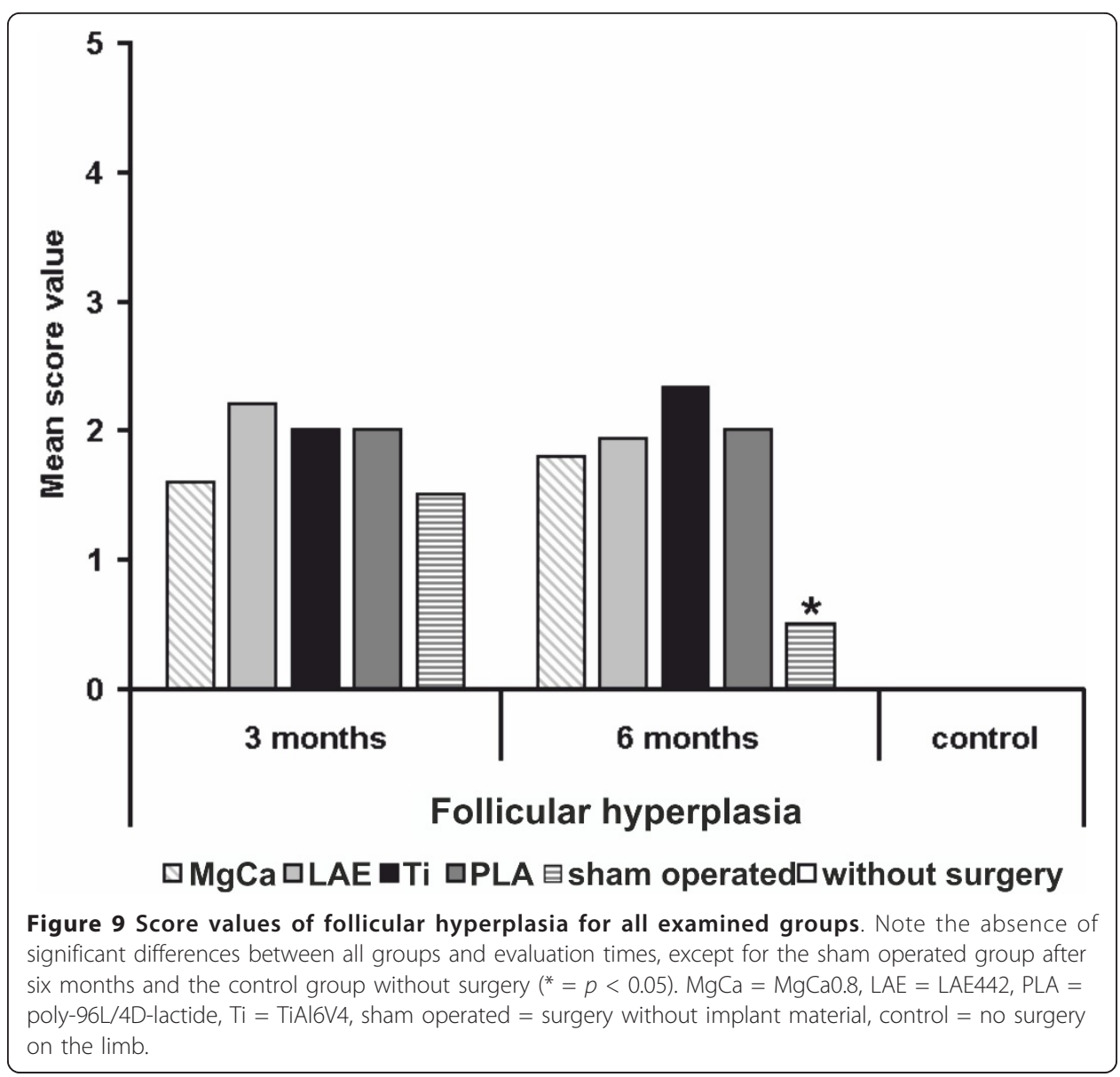

occurrence of follicular hyperplasia, its relatively low range and absence of significant differences between the control groups without implant and investigated material groups allow the conclusion of an absence of antigen features in all investigated implant materials. Moreover, the visual differences between the groups for the parameters sinus histiocytosis and heterophilic infiltration lead to the hypothesis, that the immune response to the investigated implants predominantly is a foreign-body reaction [5], which is substantiated in our study by the development of a chronic non-specific lymphadenitis. No significant differences for CD3 expression between all groups and times may also indicate the non-specific character of immune response to investigated materials. In consideration of the fact that heterophils or neutrophils as well as histiocytes take part in scavenging degradation microparticles [33], the presence of non-stained intracellular inclusions in abundant histiocytes in our study seem to be obvious reactions. Similar intracellular inclusions were also reported in other studies $[5,31,41]$.

As described in previous literature, titanium implants used in the treatment of fractures caused an immuno-inflammatory reaction in adjacent soft tissue [42,43]. These studies showed that a marked inflammation was present in the soft tissue covering the titanium implants [42] and that titanium particles were included in the cytoplasm of macrophages [43]. Furthermore, the oxidation products of titanium, especially titanium dioxide, can cause increase of apoptosis in vivo [19] and in vitro [20]. Meng et al. 
reported the cytotoxic effect of titanium microparticles on the bone marrow stem cells adjacent to the titanium implant due to phagocytosis of the particles by these cells, following the increased expression of tumor suppressor protein p53 [23]. Phagocytized microparticles of PLA also can induce apoptosis of macrophages in vivo [22]. Recent studies described the short-term $(24 \mathrm{~h})$ concentration-depended apoptotic-stimulating effects of $\mathrm{Mg}^{2+-}$ - and $\mathrm{Ca}^{2+}$ - ions as well as some rare-earth metals on cell lines [28]. However no data about the long-term apoptotic effects have been found in available literature. Dribben et al. reported on the increase of apoptosis in fetal mouse neurons under the influence of high-concentrated magnesium-ions [29]. Evidently, appearance of macrophage apoptosis could be induced by external agents, like products of degradation and oxidation of implants $[19,20]$. Therefore, the same properties could be suggested for the magnesium alloys examined in this study, as well as for the other materials. This thesis is also supported by a study of Sun and Cohen which showed a role of free calcium and magnesium ions in the early apoptotic degradation of DNA by activation of $\mathrm{Mg}^{2+} / \mathrm{Ca}^{2+}$-dependent endonuclease [30]. Theoretically, the fact of $\mathrm{Mg}^{2}$ ${ }^{+} / \mathrm{Ca}^{2+}$-dependent endonuclease activation by free $\mathrm{Mg}^{2+}$ - and $\mathrm{Ca}^{2+}$ - ions [30] as well as the fact of p53-level increase by titanium microparticles [23] allow to assume the hypothesis that titanium microparticles, free magnesium- and calcium-ions seem to induce the apoptosis similarly when DNA is injured. DNA damage leads to the accumulation of the p53 protein in cells. It first arrests the cell cycle (at the $G_{1}$ phase) to allow time for repair [33]. However, if the damage is too great to be repaired successfully, p53 triggers apoptosis, mainly by activating sensors that ultimately activate Bax and $B a k$, and by stimulating synthesis of pro-apoptotic members of the $B c l-2$ family [33]. Nonetheless, the exact pathway of apoptosis development in histiocytes in the present study remains unclear and has to be investigated in further studies.

Statistically significant differences between implantation periods could only be found for sinus histiocytosis in the titanium groups and for apoptotic histiocytes in the $\mathrm{MgCa}$ 0.8-groups. First of all, the sinus histiocytosis level after implantation of titanium implants decreased from three to six months, which may be caused by the decrease of monocytic migration to the implant surface due to progressive ossification and maturation of fibrous tissue around this non-resorbable implant [5].

Due to the ambiguity of exact apoptotic mechanism, the increase of the score value for apoptotic histiocytes in the $\mathrm{MgCa} 0.8$-group could not be completely explained. Hypothetically, the high degradation rate of $\mathrm{MgCa} 0.8$ [2] and the progressive release of degradation products might induce apoptosis $[29,30]$. Nevertheless, this suggestion should to be verified carefully in further studies. In experimental studies in rabbits, $\mathrm{MgCa}$ - as well as LAE442-alloys are tested with a good in-vivo biocompatibility and are described as possible implant materials for orthopaedic applications [36-38]. With respect to the practical implementation, it is important to note that LAE442 induced the lowest morphological changes with a low immunogenic potential. Hypothetically, these findings are due to its lower degradation rate in comparison to $\mathrm{MgCa} 0.8$ implants $[2,37,38]$ and therefore less influence of degradation products on the surrounding tissue.

In consideration of the presented data, it could be concluded that the evaluation of morphological changes in the regional popliteal lymph nodes is useful for the estimation of implant immunocompatibility. Immunohistochemistry was quite helpful for the 
sinus histiocytosis and follicular hyperplasia evaluation via identification of CD3, CD68 and CD79 $\alpha$ expression. Histological and immunohistochemical examination in our study demonstrated the common pathway of immune response for all investigated implants: the development of foreign-body reaction in the local area, followed by the development of chronic, non-specific lymphadenitis in the regional lymph nodes.

\section{Conclusion}

Although immunological reactions to titanium and PLA are observed, these materials were accepted as biocompatible implant materials in clinical use. Immunological reactions to $\mathrm{MgCa} 0.8$ increased during the observation period, but did not significantly exceed the reactions to titanium and PLA. LAE442 induced even lower morphological changes. For this reason, LAE442 seems to be a promising candidate as degradable implant material and $\mathrm{MgCa} 0.8$ might be acceptable as well. However, further research is required to deepen the understanding of immune response to bone implants and to clarify the pathway of apoptosis, which is induced by the degradation products of different implant materials.

List of Abbreviations

wt\%: weigth percent; LAE442: magnesium alloy with 90 wt $\%$ magnesium, 4 wt $\%$ lithium, 4 wt $\%$ aluminium and $2 w t \%$ rare earth composition metal; MgCa0.8: magnesium alloy with 99.2 wt $\%$ magnesium, 0.8 wt $\%$ calcium; SV: score value; MV : mean value.

\section{Acknowledgements}

This study is part of the collaborative research center (CRC 599, Medical University of Hannover, University of Veterinary Medicine Hannover and University of Hannover), which is sponsored by the German Research Foundation (DFG). Special thanks to Mrs Bettina Buck for performing the H\&E staining.

\section{Author details}

${ }^{1}$ Small Animal Clinic, University of Veterinary Medicine Hannover, Bünteweg 9, 30559 Hannover, Germany. ${ }^{2}$ Departement of Pathology, University of Veterinary Medicine Hannover, Bünteweg 17, 30559 Hannover, Germany. ${ }^{3}$ Department of Pathology, Dnipropetrovs'k State Medical Academy, str. Zhovtneva ploshcha 14, 49005 Dnipropetrovs'k, Ukraine. ${ }^{4}$ Clinic for Small Animal Surgery and Reproduction, Centre of Clinical Veterinary Medicine, Faculty of Veterinary Medicine Ludwig-Maximilians-Universität München, Veterinärstr. 13, 80539 München, Germany.

\section{Authors' contributions}

$A B$ performed the histological and immunohistochemical examinations, analysed the data and wrote the manuscript. MHT supervised the histological and immunohistochemical examinations. NE participated in the histological and immunohistochemical examinations and helped to draft the manuscript. NA participated in the animal experiment and helped to draft the manuscript. JR participated in the animal experiment and helped to draft the manuscript. AML initiated and conceived of the study, participated in its design and coordination and participated in the animal experiment. All authors read and approved the final manuscript.

\section{Competing interests}

The authors declare that they have no competing interests.

Received: 16 December 2010 Accepted: 26 April 2011 Published: 26 April 2011

References

1. Witte F, Kaese V, Haferkamp H, Switzer E, Meyer-Lindenberg A, Wirth CJ, Windhagen H: In vivo corrosion of four magnesium alloys and the associated bone response. Biomaterials 2005, 26:3557-3563.

2. Krause A, von der Höh N, Bormann D, Krause C, Bach F, Windhagen H, Meyer-Lindenberg A: Degradation behaviour and mechanical properties of magnesium implants in rabbit tibiae. J Mater Sci 2010, 45:624-632.

3. Witte F, Abeln I, Switzer E, Kaese V, Meyer-Lindenberg A, Windhagen H: Evaluation of the skin sensitizing potential of biodegradable magnesium alloys. J Biomed Mater Res A 2007, 86:1041-1047.

4. Shive MS, Anderson JM: Biodegradation and biocompatibility of PLA and PLGA microspheres. Adv Drug Deliv Rev 1997, 28:5-24.

5. Witte F, Ulrich H, Rudert M, Willbold E: Biodegradable magnesium scaffolds: Part 1: appropriate inflammatory response. J Biomed Mater Res A 2007, 81:748-756.

6. Thomann M, Krause C, Bormann D, von der Höh N, Windhagen H, Meyer-Lindenberg A: Comparison of the resorbable magnesium alloys LAE442 und MgCa0.8 concerning their mechanical properties, their progress of degradation and the bone-implant-contact after 12 months implantation duration in a rabbit model. Mat-wiss $u$ Werkstofftech 2009, 40:82-87. 
7. Peuster M, Wohlsein P, Brügmann M, Ehlerding M, Seidler K, Fink C, Brauer H, Fischer A, Hausdorf G: A novel approach to temporary stenting: degradable cardiovascular stents produced from corrodible metal-results 6-18 months after implantation into New Zealand white rabbits. Heart 2001, 86:563-569.

8. Quadbeck P, Hauser R, Kümmel K, Standke G, Stephani G, Nies B, Rößler S, Wegener B: Iron Based Cellular Metals For Degradable Synthetic Bone Replacement. PM2010 World Congress, Florenz, Italy; 2010.

9. Hallab N, Jacobs J, Black J: Hypersensitivity associated with metallic biomaterials. Biomater Eng and Devices 2000, 1:1-7.

10. Merritt K, Rodrigo Jj: Immune response to synthetic materials. Sensitization of patients receiving orthopaedic implants. Clin Orthop Relat Res 1996, 326:71-79.

11. Griem P, Gleichmann E: Metal ion induced autoimmunity. Current Opinion in Immunology 1995, 7:831-838.

12. Griem P, von Vultée C, Panthel K, Best SL, Sadler PJ, Shaw CF: T cell cross-reactivity to heavy metals: identical cryptic peptides may be presented from protein exposed to different metals. Eur J Immunol 1998, 28:1941-1947.

13. Kubicka-Muranyi M, Griem P, Lübben B, Rottman N, Lührmann R, Beyer KG: Mercuric chloride-induced autoimmunity in mice involves an upregulated presentation of altered and unaltered nucleolar self antigen. Int Arch Allergy Immunol 1995, 108:1-10.

14. Yang J, Merritt K: Production of monoclonal antibodies to study corrosion products of CO-CR biomaterials. J Biomed Mater Res 1996, 31:71-80.

15. Gil-Albarova J, Laclériga A, Barrios C, Cañadell J: Lymphocyte response to polymethylmethacrylate in loose total hip prostheses. J Bone Joint Surg Br 1992, 74:825-830.

16. Angle CR: Organ-specific therapeutic intervention. Metal toxicology Goyer RA, Klaasen CD. San Diego: Academic Press; 1995, 71-110.

17. Lalor PA, Revell PA, Gray AB, Wright S, Railton GT, Freeman MA: Sensitivity to titanium. A cause of implant failure? $J$ Bone Joint Surg Br 1991, 73:25-28.

18. Parker AW, Drez D Jr, Jacobs JJ: Titanium dermatitis after failure of a metal-backed patellas. Am J Knee Surg 1993, 6:129-131.

19. Wang L, Mao J, Zhang G, Tu M: Nano-cerium-element-doped titanium dioxide induces apoptosis of Bel 7402 human hepatoma cells in the presence of visible light. World J Gastroenterol 2007, 13:4011-4014.

20. Rahman Q, Lohani M, Dopp E, Pemsel H, Jonas L, Weiss DG, Schiffmann D: Evidence that ultrafine titanium dioxide induces micronuclei and apoptosis in Syrian hamster embryo fibroblasts. Environ Health Perspect 2002, 110:797-800.

21. Bursch W, Taper HS, Lauer B, Schulte-Hermann R: Quantitative histological and histochemical studies on the occurrence and stages of controlled cell death (apoptosis) during regression of rat liver hyperplasia. Virchows Arch, B, Cell Pathol 1985, 50:153-166.

22. Lam KH, Schakenraad JM, Esselbrugge H, Feijen J, Nieuwenhuis P: The effect of phagocytosis of poly(L-lactic acid) fragments on cellular morphology and viability. J Biomed Mater Res 1993, 27:1569-1577.

23. Meng B, Chen J, Guo D, Ye Q, Liang X: The effect of titanium particles on rat bone marrow stem cells in vitro. Toxicol Mech Methods 2009, 19:552-558.

24. Hori N, Att W, Ueno T, Sato N, Yamada M, Saruwatari L, Suzuki T, Ogawa T: Age-dependent degradation of the protein adsorption capacity of titanium. J Dent Res 2009, 88:663-667.

25. Black J: Systemic effects of biomaterials. Biomaterials 1984, 5:11-18.

26. Jacobs JJ: Corrosion of metallic implants. In Advances in operative orthopaedics. Volume 1. Stauffer, RN. St. Louis: CV Mosby; 1994:279-319.

27. Yang J, Black J: Competitive binding of chromium, cobalt and nickel to serum proteins. Biomaterials 1994, $15: 262-268$.

28. Feyerabend F, Fischer J, Holtz J, Witte F, Willumeit R, Drücker H, Vogt C, Hort N: Evaluation of short-term effects of rare earth and other elements used in magnesium alloys on primary cells and cell lines. Acta Biomater 2010, 6:1834-1842.

29. Dribben WH, Creeley CE, Wang HH, Smith DJ, Farber NB, Olney JW: High dose magnesium sulfate exposure induces apoptotic cell death in the developing neonatal mouse brain. Neonatology 2009, 96:23-32.

30. Sun $\mathrm{XM}$, Cohen $\mathrm{GM}: \mathrm{Mg}(2+)$-dependent cleavage of DNA into kilobase pair fragments is responsible for the initial degradation of DNA in apoptosis. J Biol Chem 1994, 269:14857-14860.

31. Verheyen CC, de WR, van BA, Rozing PM, de Groot K: Examination of efferent lymph nodes after 2 years of transcortical implantation of poly(L-lactide) containing plugs: a case report. J Biomed Mater Res 1993, 27:1115-1118.

32. Ferreira ME, de Lourdes Pereira M, Garcia e Costa F, Sousa JP, de Carvalho GS: Comparative study of metallic biomaterials toxicity: a histochemical and immunohistochemical demonstration in mouse spleen. $J$ Trace Elem Med Biol 2003, 17:45-49.

33. Kumar V, Abbas AK, Fausto N: Robbins and Cotran pathologic basis of disease. Elsevier/Saunders; 2004.

34. Albores-Saavedra JM, Vuitch FM, Delgado RM, Wiley EM, Hagler H: Sinus Histiocytosis of Pelvic Lymph Nodes after Hip Replacement: A Histiocytic Proliferation Induced by Cobalt-Chromium and Titanium. Am J Surg Pathol 1994, 18:83-90.

35. Munichor M, Cohen H, Volpin G, Kerner H, lancu TC: Chromium-induced lymph node histiocytic proliferation after hip replacement. A case report. Acta Cytol 2003, 47:270-274.

36. Erdmann N, Bondarenko A, Hewicker-Trautwein M, Angrisani N, Reifenrath J, Lucas A, Meyer-Lindenberg A: Evaluation of the soft tissue biocompatibility of $\mathrm{MgCa} 0.8$ and surgical steel $316 \mathrm{~L}$ in vivo: a comparative study in rabbits. Biomed Eng Online 2010, 9:63.

37. Reifenrath J, Krause A, Bormann D, von Rechenberg B, Windhagen H, Meyer-Lindenberg A: Profound differences in the in-vivo-degradation and biocompatibility of two very similar rare-earth containing $\mathrm{Mg}$-alloys in a rabbit model. Mat-wiss u Werkstofftech 2010, 41:1054-1061.

38. Li Z, Gu X, Lou S, Zheng Y: The development of binary Mg-Ca alloys for use as biodegradable materials within bone. Biomaterials 2008, 29:1329-1344.

39. Jones $M$, Cordell JL, Beyers AD, Tse AG, Mason DY: Detection of $T$ and $B$ cells in many animal species using crossreactive anti-peptide antibodies. J Immunol 1993, 150:5429-5435. 
40. Lee S, Goh BT, Lai SH, Tideman H, Stoelinga PJ, Jansen JA: Peri-implant and systemic release of metallic elements following insertion of a mandibular modular endoprosthesis in Macaca fascicularis. Acta Biomater 2009, 5:3640-3646.

41. Krause A: Untersuchung der Degradation und Biokompatibilität von degradablen, intramedullären Implantaten auf Magnesiumbasis im Kaninchenmodell. Dissertation Hannover: Tierärztliche Hochschule; 2008.

42. Voggenreiter G, Leiting S, Brauer H, Leiting P, Majetschak M, Bardenheuer M, Obertacke U: Immuno-inflammatory tissue reaction to stainless-steel and titanium plates used for internal fixation of long bones. Biomaterials 2003, 24:247-254.

43. Katou F, Andoh N, Motegi K, Nagura H: Immuno-inflammatory responses in the tissue adjacent to titanium miniplates used in the treatment of mandibular fractures. J Craniomaxillofac Surg 1996, 24:155-162.

doi:10.1186/1475-925X-10-32

Cite this article as: Bondarenko et al:: Comparison of morphological changes in efferent lymph nodes after implantation of resorbable and non-resorbable implants in rabbits. BioMedical Engineering OnLine 2011 10:32.

Submit your next manuscript to BioMed Central and take full advantage of:

- Convenient online submission

- Thorough peer review

- No space constraints or color figure charges

- Immediate publication on acceptance

- Inclusion in PubMed, CAS, Scopus and Google Scholar

- Research which is freely available for redistribution 